\title{
ÉTICA, ESTÉTICA Y CIUDAD (VIVIBLE E INVIVIBLE)
}

Francisco Iracheta*

RESUMEN: Se examina la relación que tiene la ética con la estética desde una perspectiva filosófica y la aplicación que tiene la relación de una con la otra en el contexto del concepto de ciudad vivible. Utilizando las categorías de "estabilidad" y "cultura y medio ambiente" que la revista The Economist ha venido usando para medir la habitabilidad de las ciudades, se reflexiona en torno a la idea de que cualquier ciudad que cumple indicadores estéticos, cumple también indicadores éticos.

$$
\text { শ্ৰ) }
$$

\section{ETHICS, Aesthetics AND THE City (LIVABLE AND UNLIVABLE)}

ABSTRACT: This article discusses the relationship between ethics and aesthetics from a philosophical perspective and the application of this relationship in the context of the concept of a livable city. Using the categories of "stability" and "culture and environment" that The Economist magazine has been employing to measure the liveability of cities, the article reflects on the idea that any city that addresses aesthetic indicators in a positive way also addresses ethical indicators.

PALABRAS CLAVE: ciudades vivibles, estabilidad, medio ambiente.

KEY WORDS: liveable cities, stability, environment.

RECEPCIÓN: 25 de enero de 2018.

APROBACIÓN: 11 de julio de 2018.

* Instituto Tecnológico y de Estudios Superiores de Monterrey, campus Puebla. 
Se prohíbe su reproducción total o parcial por cualquier medio, incluido electrónico, sin permiso previo y por escrito de los editores. 


\section{ÉTICA, ESTÉTICA Y CIUDAD (VIVIBLE E INVIVIBLE)}

1. Al final del Tractatus LogicoPhilosophicus, el filósofo austriaco Ludwig Wittgenstein señaló que "la ética y la estética son la misma cosa". ${ }^{1}$ El concepto de bien moral y el de lo bello pertenecen a una misma realidad. Se trata de una idea que en el contexto de la filosofía del primer Wittgenstein resulta lógica, dado el tipo de estructura lingüística que, de acuerdo con su análisis, poseen los juicios normativos sobre el bien y lo bello. Los juicios de la ética y la estética (pues aquí no se puede hablar de "proposiciones") carecen de significado propiamente porque traspasan el umbral de los hechos, de todo aquello que "es el caso", para situarse en lo que trasciende el mundo fáctico y la experiencia.

No obstante, si bien la idea de emparentar ética y estética parte del punto de vista de una filosofía del lenguaje que básicamente separa todo lo que es decible claramente en relación con los hechos de lo que no lo es, también es cierto que se trata de una asociación de ideas tan vieja como la propia filosofía sistemática.

Atiéndase, en efecto, como segundo botón de muestra, el siguiente pasaje del Banquete, de Platón:

[D]ebe considerarse más valiosa la belleza de las almas que la del cuerpo, de suerte que, si alguien es virtuoso de alma, aunque tenga un escaso

${ }^{1}$ Ludwig Wittgenstein, Tractatus Logico-Philosophicus, 1999, Nueva York, Routledge, p. 183. 
esplendor, séale suficiente para amarle, cuidarle, engendrar y buscar razonamientos que hagan mejores a los jóvenes, para que sea obligado, una vez más, a contemplar la belleza que reside en las normas de conducta $y$ en las leyes y reconocer que todo lo bello está emparentado consigo mismo. $^{2}$

Platón hace alusión a "bellas” almas que lo son en razón de las virtudes que poseen (como rasgos de carácter), de modo que, necesariamente, un alma que, por ejemplo, es justa, es un alma bella. También, como puede verse, Platón hace una relación entre la belleza de las almas y el seguimiento de las normas de conducta y las leyes, las cuales son políticas (cívicas) por su propia naturaleza. Aunque Wittgenstein no tiene por qué compartir el sistema metafísico de la filosofía platónica y seguramente Platón no hubiera compartido la idea de Wittgenstein sobre la necesidad de guardar silencio ante manifestaciones éticas y estéticas porque arremeten contra los límites del lenguaje, nuestros dos filósofos sí comparten la idea según la cual entre lo bello y lo bueno existe una compenetración indisociable; y nuevamente, si bien los juicios éticos y estéticos no pueden ser aclarados con una sola observación empírica de los hechos, ambos también estarían de acuerdo en que las ideas de la ética y la estética (y, tal vez, añadiríamos las religiosas) son las que dan sentido a la existencia humana, y no las categorías científicas que solo se reducen a mostrar tautológicamente la realidad tal cual es. Escribió Wittgenstein en el Tractatus: "sentimos que aun cuando todas las cuestiones científicas hayan recibido respuesta, nuestros problemas vitales todavía no se han rozado en lo más mínimo".3

Desde luego, y quizás más que las dos filosofías anteriores, la alusión a Kant no puede faltar cuando asociamos ideas éticas y estéticas. En su filosofía trascendental, los juicios éticos y estéticos se definen como juicios reflexivos, pues tanto uno como otro tienen la función de determinar el particular para el universal dado (que es el bien y lo bello). Uno y otro tipo de juicios normativos, como juicios que se sostienen

${ }_{2}^{2}$ Platón, Diálogos, 2000, Madrid, Gredos, p. 260. Las cursivas son mías.

${ }^{3}$ Wittgenstein, Tractatus, p. 187. 
desde la razón, nunca son privados, sino que más bien siempre son de naturaleza social (pública). Están guiados — dice Kant — por la máxima de la razón del pensamiento intersubjetivo: "pensar siempre poniéndose en el lugar de los demás". Esta máxima aparece en la discusión sobre el "gusto como un tipo de sentido compartido" (sensus communis) en la tercera Crítica. Kant explica que el signo de cualquiera que tiene un pensamiento ampliado está en que "deja a un lado las condiciones subjetivas de su propio juicio, en el cual muchos se encuentran confinados, y reflexiona desde un punto de vista universal". ${ }^{4}$ Así, nuestros propios pensamientos estéticos devienen objetivos y podemos cumplir, por tanto, con una de las reglas que garantizan la objetividad del gusto. Más aún, en sus reflexiones antropológicas, Kant se refiere a este principio como la "piedra de toque del sano" entendimiento:

Pues es una piedra de toque subjetivamente necesaria de la rectitud de nuestros juicios en general y, por lo mismo, de la sanidad de nuestro entendimiento, el que lo confrontemos con el entendimiento ajeno, y no nos aislemos con el nuestro y, por decirlo así, juzguemos públicamente con nuestra representación privada. ${ }^{5}$

Cualquier juicio que no tome en consideración el pensamiento de los demás, o lo que es lo mismo, "no se juzgue en un mundo común con los demás”, sino que se asuma que las propias representaciones son las propias representaciones de los demás, será un juicio meramente subjetivo, "con el cual uno es inducido a engañarse a sí mismo". ${ }^{6}$ En el mismo texto de la Antropología, Kant trata el principio del pensamiento intersubjetivo como la máxima de "imaginarse (al comunicarse con los demás) en el lugar del otro", el cual también denomina "liberal". Finalmente, en su exposición de "la armonía de la política con la moral", en el "Apéndice" de la Paz perpetua, el filósofo de Königsberg vuelve

${ }^{4}$ Immanuel Kant, Critique of judgment, 1951, Nueva York, Hafner Library of Classics, p. 137.

${ }^{5}$ Kant, Antropología, 2004, Madrid, Alianza, pp. 141-142.

${ }^{6}$ Loc. cit. 
a hacer alusión al principio de la publicidad tal como aparece en la discusión del gusto estético en la tercera Crítica, pero ahora formulado como juicio de la razón objetiva práctica: "son injustas todas las acciones que se refieran al derecho de otros hombres cuyos principios no soportan ser publicados". ${ }^{7}$ Se trata, nos dice Kant, de un principio tanto ético como jurídico, "pues pertenece a la doctrina de la virtud y al derecho de los hombres". 8

2. En años recientes y hasta la fecha, la Unidad de Inteligencia de la revista semanal inglesa The Economist (EIU) publica un estudio sobre las ciudades más habitables del planeta. Considerada como "líder en la inteligencia global de los negocios", la EIU tiene la reputación de ser un "ideal comentador, interprete y pronosticador" de sucesos económicos, políticos y sociales de trascendencia que ocurren en al menos 140 países, situados dentro del contexto de la globalización. En el estudio anual de libre acceso en línea "The global liveability report", 9 se hace una clasificación de las 10 ciudades que ofrecen mejores condiciones de vida y de las 10 que mantienen las peores.

En el informe se señala que "el concepto de habitabilidad (liveability) es simple: evalúa qué lugares proveen las mejores o peores condiciones de vida". ${ }^{10}$ El concepto tiene varios propósitos, aunque fundamentalmente económicos: brindar a inversores y tomadores de decisiones de negocios información valiosa sobre el estado de vida de las ciudades en las cuales piensan o quieren invertir. Para realizar el estudio se utilizan cinco variables que contienen en conjunto treinta indicadores cualitativos y cuantitativos. ${ }^{11}$

${ }^{7}$ Kant, Sobre la paz perpetua, 2002, Madrid, Alianza, p. 100.

${ }^{8}$ Loc. cit.

${ }^{9}$ The Economist Intelligence Unit, “The global liveability report 2017”, Londres, 2017, The Economist Intelligence Unit Limited, en $<$ http://www.eiu.com/Handlers/WhitepaperHandler. ashx?fi=Liveability-Ranking-Free-Summary-Report-August-2017.pdf\&mode=wp\&campaignid $=$ Liveability $17>$, consultado el 25 de enero de 2018 .

${ }^{10}$ Ibid., p. 7.

${ }^{11}$ Ibid., pp 8-9. 
Los indicadores de cada variable se aplican a cada ciudad evaluada, y de ahí se obtiene una calificación que oscila entre "aceptable", "tolerable", "inconfortable", "indeseable" e "intolerable". La escala va del 1 al 100, 1 es "intolerable" y 100 el "ideal" de lo "aceptable". Además, cada categoría tiene un porcentaje $/ 100 \%$, de modo que la categoría de "estabilidad" tiene el porcentaje más alto, empatada con la categoría de "cultura y medioambiente" con $25 \%$, y la de educación, con $10 \%$, es la más baja.

No es fácil estar de acuerdo en que la categoría de "educación" merece la calificación más baja, pues, si como repetimos hasta el cansancio, la educación es la respuesta para casi todos los problemas humanamente controlables, entonces intuitivamente tendría que estar entre las categorías con los porcentajes más altos. Pero para mis propósitos inmediatos, me interesa más subrayar que las categorías de estabilidad de cultura y medioambiente, las más altas de las cinco, contienen indicadores de habitabilidad que tienen sentido por sus significados éticos y estéticos, respectivamente. Al menos, reconocemos que no hay derechos justos asentados en costumbres irracionales e indeseables, $\mathrm{y}$ que es difícil sentir gusto y reconocer la belleza de cualquier cosa que pueda ocurrir dentro de un cuadro de desolación y barbarie provocado por la violencia humana.

La categoría de "estabilidad" se refiere a situaciones sociopolíticas estables dentro de las ciudades, o para ponerlo negativamente, situaciones que no son delincuenciales, de terror o conflicto. Así, una ciudad inestable es una ciudad violenta. La gravedad de toda acción delictiva, por atentar contra el derecho a la vida o la propiedad de las personas, se mide por sus referencias a conceptos éticos fundamentales, como la libertad, la justicia o la dignidad; y, por otro lado, los indicadores de "cultura disponible" y de "consumo de bienes y servicios" (por citar los menos), que pertenecen a la categoría de la "cultura y medio ambiente", los asociamos fácilmente con el gusto y la apreciación por lo bello en un entorno urbano, como la vivencia de jardines y plazas públicas, parques, cielos libres de contaminación, banquetas y calles saneadas, museos que pueden ser visitados, paisajes arquitectónicos y urbanos, etcétera. 
Por séptimo año consecutivo (2011-2017), la ciudad de Melbourne, Australia, ocupa el primer lugar como la más habitable del mundo, seguida por Viena, Vancouver, Toronto y Calgary. De hecho, según el último informe (2017), estas primeras cinco ciudades no han cambiado de posición en siete años, aunque ha habido "volatilidad en sus calificaciones debido a que en los últimos tiempos se ha visto un incremento de la inestabilidad en el mundo". ${ }^{12}$

Por el otro lado, las cinco ciudades menos habitables del mundo, según los informes de 2016 y 2017, son, de menos a más, Damasco (Siria), Lagos (Nigeria), Trípoli (Libia), Dhaka (Bangladesh) y Puerto Moresby, la capital de Papúa Nueva Guinea. Todas estas ciudades mantienen cifras altísimas de inestabilidad debido a los disturbios civiles provocados por terrorismo, guerras civiles, enfrentamientos entre militares y grupos insurgentes o cárteles de drogas, entre otros.

Existen interesantes cambios percentiles en todas estas 10 ciudades entre uno y otro informe (2016-2017). Por ello se sugiere al lector interesado descargar los documentos completos. Me basta con decir aquí que se remarca en uno y otro que la presencia del conflicto es el factor clave que explica la caída de los niveles de habitabilidad en las ciudades.

3. Yo creo que la noción de "ciudades habitables" se justifica con el uso de parámetros de medición que brindan un buen ejemplo práctico de lo que Platón, Kant y Wittgenstein piensan acerca de la estrecha relación que guardan la ética y la estética. Al menos si admitimos la relación causal que una de ellas, la ética, puede tener sobre la otra, la estética, y asentimos además a que los juicios de gusto sobre la vida urbana cuentan como juicios estéticos, así como los problemas de violencia civiles o de corrupción política e impunidad en los sistemas de justicia cuentan como problemas éticos. Por supuesto, además, no quiero decir que se trata del único ejemplo, ni es tampoco el más paradigmático de lo que esta relación significa, a juzgar por lo que estas tres personalidades filosóficas aseveran. Cada uno de estos tres sistemas de pensamiento está

${ }^{12}$ Ibid., p. 4 
constituido por ideas y presupuestos metafísicos que no permiten con facilidad hallar sus correlatos, sobre todo éticos, en el mundo de los hechos y la experiencia. Esta es una de las razones por la cuales resulta sumamente arriesgado sostener que la intención que perseguían estos tres filósofos con la formulación teórica de sus sistemas era llevarnos a concluir lógicamente que todo esfuerzo humano tiene que desembocar por fuerza en hacer de nuestras ciudades entornos vivibles bellos y justos. Sin embargo, dada la general aceptación o universal reconocimiento de las ideas de lo bueno y de lo bello y del estrecho vínculo que tienen estas ideas entre sí como consecuencia de la enorme influencia que ha tenido el pensamiento filosófico en la educación y en la formación y propagación de la cultura, parece natural reconocer que, en las ciudades, como los espacios habitables con mayor presencia educativa y cultural en el mundo, los conceptos éticos y estéticos se han hecho vivenciales más allá de sus confines meramente teóricos. Muchas disciplinas han contribuido a fomentar una conciencia común o pensamiento generalizado por hacer más vivibles, ética y estéticamente, nuestros espacios comunes y formas compartidas de vida. Las artes plásticas como la arquitectura y la escultura, así como el urbanismo y las políticas públicas (dentro del contexto de la gobernanza), han contribuido enormemente a hacer práctico esto que nuestras tres figuras filosóficas pensaron echando mano de todo un andamiaje metafísico trascendental que estas disciplinas, evidentemente, obvian en su propio ejercicio. Basta centrar la atención en todos los espacios que existen en cualquier ciudad, en grados cuantitativos diversos, que combinan creatividad arquitectónica con una adecuada e inteligente planeación urbanista y que se hallan enmarcados dentro de un contexto sociopolítico que de manera clara y verdadera manifiesta un gradual abatimiento de prácticas viciosas en la vida colectiva y un gradual fortalecimiento de virtudes cívicas en los ciudadanos y en los funcionarios públicos. Cuando existe armonía entre espacios construidos con buen gusto y sanas políticas públicas, el resultado no es otro sino la convivencia ciudadana que promueve eso que Aristóteles llamaba en su ética la amistad cívica, ${ }^{13}$ la cual, sin duda alguna, es una condición necesaria para la solidaridad.

${ }^{13}$ Aristóteles, Ética nicomáquea, 1985, Madrid, Gredos, p. 363. 
A juzgar por la importancia que tiene la idea de vivir en ciudades justas, limpias y bellas, me parece que vivir la ciudad aparece como un rasgo evaluativo de peso para decidir vivir en una ciudad.

4. Tenemos entonces que hay varios indicadores que sirven como criterios de medición para determinar la calidad de vida en las ciudades. Estos indicadores inciden directamente en lo que tiene que ver con el gusto y el sentido estético de las ciudades, y con la conciencia y prácticas de cooperación ciudadana, la paz social y el comportamiento de gobernantes e instituciones de gobierno, elementos todos asociadas a la ética, tanto teórica como aplicada, profesional y cívica. Estamos hablando, recordemos, de las categorías de "estabilidad" y de "cultura y medioambiente". Ahora, para terminar esta reflexión quisiera llamar la atención a la manera en que el informe señala la relación entre sí de estos indicadores, pues muestra en la práctica lo que Platón, Kant y Wittgenstein manifiestan en sus sistemas filosóficos sobre la relación entre ética y estética.

En primer lugar, hay que decir que los indicadores, tal como se manejan en el informe, sirven a modo de patrones revisores o evaluadores de sucesos que ocurren en cualquier organización social, dentro de un marco intencional definido y mínimamente compartido entre las partes que la conforman e integran. Los indicadores ayudan a explicar el comportamiento de cualquier organización social y a entender su avance o retroceso, lo que es fundamental para poder gobernarla. A su vez, el comportamiento se mide con una meta o resultado con suficiente valor objetivo, esto es, un fin, por lo que el indicador, para poder ser una guía lo más certera posible, requiere medir según una cuota de valor compartida. Si seguimos lo que, grosso modo, dictan las filosofías trascendentales de Platón, Kant y Wittgenstein, diríamos que los indicadores se encuentran a mitad de camino entre el arquetipo y los hechos; entre el ideal que filosóficamente puede ser solo capturado o aprehendido intelectualmente de forma a priori, independientemente de la experiencia, y lo que la experiencia muestra como hechos observables y cuantitativamente medibles, esto es, aprehendidos a posteriori. 
Con todo, desde el punto de vista de un sano sentido común pragmático podemos decir que, fuera de la filosofía que estas tres figuras inmortales del pensamiento hicieron suya en su momento histórico, no estamos obligados a recorrer este camino de fundamentación metafísica. Se puede plantear de manera inversa, a saber: argumentando que las filosofías de Platón, Kant y el primer Wittgenstein quieren explicar, en lenguaje metafísico, lo que de manera común aceptamos sin necesidad de tantos oscuros rodeos ni de un determinado compromiso escolástico. En este sentido, decimos que los indicadores sirven porque la observación de los hechos nunca está libre de una interpretación normativa como la que dan los valores éticos y estéticos y, a la vez, ninguna interpretación normativa como la que comparten la ética y la estética tiene sentido a menos que esté anclada en los hechos. Por ello, desde esta perspectiva no parece tener mucho sentido hablar de hechos o de normatividad ética y estética sin más. La filosofía - la metafísica, en particular - es la que llega después a enmudecer las aguas, para seguir nuevamente con Wittgenstein, pero no ya del Tractatus.

Sea como sea que tratemos a los indicadores - bien como aplicaciones de la metafísica a la experiencia mundana o como las escaleras por las que sube la metafísica para después tirarla por la borda- podemos decir que hay un acuerdo en que el fin ideal que sirve como parámetro de medición de los acontecimientos observables en un entorno social no es comprensible sin valores éticos y estéticos. Esta relación aparece de manera "natural" en la tabla que señala las mejores y peores ciudades para vivir: no hay ninguna de ellas en donde las categorías de "estabilidad" y "cultura y medio ambiente" no mantengan un promedio porcentual similar; y también la relación se mantiene al señalar que el deterioro de lo que mide el primer indicador es causante de un deterioro de lo que mide el tercero. Las disrupciones en el tejido social son responsables de que se causen daños en otros ámbitos relacionados con manifestaciones estéticas. En efecto, como podemos leer en el estudio:

El conflicto es responsable de muchas de las calificaciones más bajas en las clasificaciones. Esto no solamente se debe a que los indicadores de estabi- 
lidad tienen, individualmente, las mediciones más altas, sino también porque los factores que definen la estabilidad en las ciudades se expanden y tienen un efecto adverso en otras categorías. Por ejemplo, el conflicto no solo es causa de la disrupción en sí misma, sino también daña la infraestructura, al sobrecargar los hospitales y menguar la disponibilidad de bienes, servicios y actividades recreacionales. ${ }^{14}$

Una ciudad en continuo conflicto (social, político, económico o religioso), con brotes continuos de violencia, genera al mismo tiempo problemas urbanos y culturales que impactan drásticamente en la vivencia estética. Piénsese, por ejemplo, en el caso trágico de Damasco, la ciudad que aparece en último lugar de las 140 investigadas según el informe de la EIU. Debido a su historia de más de 4000 años, que le ha valido ser reconocida por la UNESCO como patrimonio universal de la humanidad, Damasco ha sido durante siglos una ciudad con gran riqueza cultural y artística y por ello visitada por millones de personas. Sin embargo, a raíz de la violencia desencadenada por su guerra civil, que ha cobrado la vida de más de 320000 personas y ha obligado a otros tantos cientos de miles a emigrar del país (en violación a los derechos humanos), la ciudad ha sufrido un abandono de sus áreas antiguas, cuyos daños arquitectónicos son ahora invaluables y en muchos casos irreversibles. El testimonio del periodista de The New York Times, Somini Sengupta, es revelador:

Un comerciante de arte, Samer Kozah, vive en la antigua casa de su familia, rodeado de obras de artistas que han huido. Le pregunté cómo había cambiado la ciudad: una pregunta que me pareció inofensiva, pero que lo hizo romper en llanto. Su esposa abandonó el patio donde estábamos sentados. ${ }^{15}$

También en el informe de 2017 se alude a que la llegada de Donald Trump a la presidencia, junto con otras prácticas de violencia injus-

${ }^{14}$ The Economist Intelligence Unit, p. 5.

${ }^{15}$ Somini Sengupta, "Damasco, la ciudad solitaria", The New York Times, 26 de noviembre de 2017, en <https:/www.nytimes.com/es/2017/11/26/damasco-siria-guerra-civil-destruccion/>, consultado el 20 de enero de 2018. 
tificada de la policía en contra de personas negras, ha provocado en Estados Unidos disturbios y marcadas divisiones sociales:

En los años recientes, algunas ciudades en Estados Unidos han registrado un declive en sus calificaciones como ciudades habitables. En parte, se debe a los disturbios relacionados con la muerte de afroamericanos a manos de agentes de policía. Por añadidura, se han realizado protestas constantes contra las órdenes políticas y ejecutivas del presidente Donald Trump. ${ }^{16}$

Además de que el gobierno de Trump no ha hecho casi nada por revertir este grave problema racial, ha ocasionado problemas en la vivencia de espacios públicos y represión de diversas manifestaciones estético-sociales, relacionadas con proyectos asociados a los Dreamers, inmigrantes, mexicanos, grupos feministas y la comunidad gay-lésbica, por mencionar solo unos pocos. Pero es más difundido el impacto que la llegada de Trump ha tenido en asuntos específicos de políticas medioambientales. La categoría de "cultura y medio ambiente" incluye indicadores que miden condiciones ambientales y climáticas, y al quedar demostrada la relación que existe entre débiles políticas públicas en materia de contaminación ambiental y deterioro climático, resulta una ofensa para cualquier ciudadano sensible que Donald Trump no esté dispuesto a hacer nada para revertir el cambio climático mediante una concienzuda revisión de políticas ambientales porque, sencillamente, no cree que haya un cambio climático ocasionado por la contaminación que los seres humanos causamos. Definitivamente, el verde y el azul no son los colores de Donald Trump, ni por tanto los bellos cielos profundos que existen a espaldas de su silla en el despacho presidencial ni los jardines y parques que alguna ciudad, si acaso alguna vez en un mundo posible imaginado para él, pudiera llegar a albergar.

En este orden de ideas, es notable el contraste con lo que ocurre en materia de políticas medioambientales en la ciudad más habitable del mundo. Melbourne tiene una amplia dotación de parques y jardines públicos que brindan a los citadinos entornos de belleza natural dentro

${ }^{16}$ The Economist Intelligence Unit, p. 3. 
de la gran urbe, además, claro está, de que alimentan con oxígeno a la ciudad. En 2013, Melbourne obtuvo el certificado como ciudad neutra en carbono, al lograr contrarrestar estas emisiones mediante un proyecto que combinó políticas públicas con educación medioambiental. Inevitablemente nos vuelve a hacer pensar, para concluir este texto, en lo siguiente: el porcentaje que el estudio da a la educación, de 10\%, es realmente poco; pues la educación ética y estética es el medio más razonable para que el 50\% de valor que tienen las categorías que cubren la habitabilidad de las ciudades, en materia de "estabilidad" y "cultura y medio ambiente", se conviertan en una realidad. 